\title{
Exploratory study on application of MALDI-TOF-MS to detect serum and urine peptides related to small cell lung carcinoma
}

\author{
PANPAN LV ${ }^{1,2}$, ZEYUAN LIU $^{1}$, BIN XU $^{3}$, CHUANHAO TANG $^{1}$, XIAOYAN LI $^{1}$, HAIFENG QIN $^{1}$, \\ SHAOXING YANG ${ }^{1}$, HONGJUN GAO $^{1}, \mathrm{KUN} \mathrm{HE}^{3}$ and XIAOQING LIU ${ }^{1}$ \\ ${ }^{1}$ Department of Pulmonary Oncology, The Fifth Medical Center of Chinese PLA General Hospital, Beijing 100071; \\ ${ }^{2}$ Academy of Military Medical Science; ${ }^{3}$ National Center of Biomedical Analysis, Beijing 100069, P.R. China
}

Received December 19, 2018; Accepted June 25, 2019

DOI: $10.3892 / \mathrm{mmr} .2019 .10794$

\begin{abstract}
Matrix-assisted laser desorption/ionization time-of-flight mass spectrometry (MALDI-TOF-MS) was employed to analyze differential serum and urine peptides in patients with small cell lung cancer (SCLC) and healthy individuals, and SCLC diagnostic classification models were constructed. Serum and urine samples from 72 patients with SCLC, age- and gender-matched with 72 healthy individuals, were divided into training and testing sets in a 3:1 ratio. Serum and urine peptides were extracted using copper ion-chelating nanomagnetic beads, and mass spectra were obtained using MALDI-TOF-MS. Peptide spectra for the training set were analyzed, and the classification model was constructed using ClinProTools (CPT). The testing set was used for blinded model validation. For training-set sera, 122 differential peptide signal peaks with a mass of $0.8-10 \mathrm{kDa}$ were observed, and 19 peptides showed significantly different expression $[\mathrm{P}<0.0005$; area under curve (AUC) $\geq 0.80]$. CPT screened 5 peptide peaks $(0.8114,0.83425,1.86655,4.11133$ and $5.81192 \mathrm{kDa})$ to construct the classification model. The testing set was used for the blinded validation, which had $95.0 \%$ sensitivity and $90.0 \%$ specificity. For the training-set urine, 132 differential peptide signal peaks with $\mathrm{m} / \mathrm{z}$ ratios of $0.8-10 \mathrm{kDa}$ were observed, and 8 peptides had significantly different expression $(\mathrm{P}<0.0005$; $\mathrm{AUC} \geq 0.80)$. Then, 5 peaks $(1.0724,2.37692$, $2.7554,4.75475$ and $4.7949 \mathrm{kDa})$ were used for classification model construction. The testing set was used for 36 blinded
\end{abstract}

Correspondence to: Professor Xiaoqing Liu, Department of Pulmonary Oncology, The Fifth Medical Center of Chinese PLA General Hospital, 8 Dongjie Road, Fengtai, Beijing 100071, P.R. China E-mail: liuxiaoqing@csco.org.cn

Abbreviations: AUC, area under curve; CPT, ClinProTools; ED, Extensive Disease; HCCA, hydroxycinnamic acid; LD, Limited Disease; MALDI-TOF-MS, matrix-assisted laser desorption/ionization time-of-flight mass spectrometry; SCLC, small cell lung cancer; SPE, solid-phase extraction

Key words: MALDI-TOF-MS, proteomics, serum, small cell lung carcinoma, urine validation, which had $85.0 \%$ sensitivity and $80.0 \%$ specificity. Among the differential peptides, 3 had the same significant peaks at $2.3764,0.8778$ and $0.8616 \mathrm{kDa}$, identified as fibrinogen $\alpha$, glucose-6-phosphate isomerase and cyclin-dependent kinase-1, respectively. The present study highlighted the differences that exist in serum and urine peptides between patients with SCLC and healthy individuals. Serum and urine peptide diagnostic classification models could be constructed using MALDI-TOF-MS, and showed high sensitivity and specificity.

\section{Introduction}

Lung cancer, also known as primary bronchogenic carcinoma of the lung, is a common malignancy, the incidence of which has increased rapidly in previous years in China (1). Data published by the National Central Cancer Registry of China in 2014 showed 605,900 new cases of lung cancer in China, the highest of all malignant tumors; $~ 66 \%$ of the patients were diagnosed too late for radical surgery $(2,3)$. Of those diagnosed too late, the 5-year survival rate of patients with small cell lung cancer (SCLC), accounting for 15-17\% of all lung cancers, was only $6 \%$; however, the median 5 -year survival rate for limited-stage SCLC was $28-60 \%$ (4). Thus, early diagnosis is clearly essential for reducing SCLC mortality. Many patients undergo a delayed examination and further treatment because they exhibit no specific symptoms. Currently, traditional medical diagnosis primarily relies on imaging examinations (5); however, the number of potentially transformed cell clones in patients with early-stage lung cancer is lower than the minimum threshold of current imaging and measurement technology. Therefore, searching for sensitive and specific diagnostic methods applicable even before imaging is necessary to increase the rate of early lung cancer diagnosis.

Cancer proteomics is the study of cancer from the perspective of protein and peptide fragments, and has great potential for early cancer diagnosis. In recent years, mass spectrometry (MS) of proteins and peptides has been increasingly applied to exploratory studies on the early diagnosis of breast, stomach and colon cancers, and other malignancies (6-8). The present study employed matrix-assisted laser desorption/ionization time-of-flight (MALDI-TOF)-MS and nanomagnetic beads to extract serum peptides in combination with the ClinProt system to conduct an exploratory 
analysis of the sera and urine of patients with SCLC and healthy individuals, and screen for differentially expressed peptides for establishing a foundation for the SCLC serum and urine mass spectrometric diagnostic method. To the best of our knowledge, this is the first exploratory study to identify small cell lung cancer by blood and urine using MALDI-TOF-MS.

\section{Materials and methods}

Sample source and collection. In total, 155 patients with SCLC at the Department of Lung Cancer of The Fifth Medical Centre, Chinese PLA General Hospital, between October 2014 and April 2016, but only 72 patients with SCLC were enrolled into the study according to the admission criteria, with a median age of 58 (range, 36-83) years and a male:female ratio of 1:1.54. Enrolled patients fulfilled the following criteria: i) Histopathologically or cytologically confirmed as a patient with small cell lung carcinoma [Extensive Disease (ED) or Limited Disease (LD) stage] (9); ii) aged 18 years or older; iii) Eastern Cooperative Oncology Group Performance Status score (9) <2; and, iv) had not previously undergone chemotherapy, radiotherapy, targeted therapy or other cancer treatments. Over the corresponding period, 72 serum and urine samples were collected from healthy individuals who underwent a physical examination at the hospital and were age- and sex-matched with the patients. The median age was 56 (range, 31-72) years and the male:female ratio 1:1.32. These healthy individuals were selected after fulfilling the following criteria: Older than 18 years, and without pulmonary nodules, pneumonia, tuberculosis, or other abnormalities. This study was approved by the Ethics Committee of The Fifth Medical Centre, Chinese PLA General Hospital (no. 2012-11-171). All patients provided written informed consent before undergoing any treatment, or serum or urine sampling. The study overview is shown in Fig. S1 and basic information regarding the characteristics of patients and healthy subjects is presented in Table I.

Serum (urine) sampling procedure. During fasting, $3 \mathrm{ml}$ of peripheral venous whole blood (15 $\mathrm{ml}$ of midstream urine) was collected at 8:00 am in a test tube containing EDTA anticoagulant (urine collection tube). Samples were maintained at $21^{\circ} \mathrm{C}$ for $2 \mathrm{~h}$, then centrifuged at $2,500 \mathrm{xg}$ for $10 \mathrm{~min}$ at $4^{\circ} \mathrm{C}$. The supernatant (serum or urine) was collected, divided into $150 \mu \mathrm{l}$ aliquots, and stored in a freezer at $-80^{\circ} \mathrm{C}$.

Reagents and instrumentation. The following reagents and equipment were used in the present study: Trifluoroacetic acid (TFA, Sigma-Aldrich; Merck KGaA); acetonitrile (ACN; Thermo Fisher Scientific, Inc.); $\alpha$-Cyano-4-hydroxycinnamic acid (HCCA) and peptide mixture (Bruker Corporation); copper ion-chelating nanomagnetic beads and buffer system (National Center of Biomedical Analysis, Beijing, China); MALDI-TOF-MS instrument (Ultraflex; Bruker Corporation); magnetic bead separator (Bruker Corporation); 3K15 refrigerated benchtop centrifuge (Sigma-Aldrich; Merck KGaA); MTP Anchorchip targets (Var/384; Bruker Corporation); and ClinProTools version 3.0 analytical software (CPT; Bruker Corporation).
Sample preparation and mass analysis (peptide profiling). Serum and urine samples from 72 patients with SCLC and 72 healthy individuals were randomly divided into two groups in a 3:1 ratio as follows: A training set comprising samples from 54 patients with SCLC (lung cancer serum group I), 54 patients with SCLC (lung cancer urine group I), 54 healthy individuals (healthy serum group I), and 54 healthy individuals (healthy urine group I) was used to construct the classification model, and a testing set comprising samples from 18 patients with SCLC (lung cancer serum group II), 18 patients with SCLC (lung cancer urine group II), 18 healthy individuals (healthy serum group II), and 18 healthy individuals (healthy urine group II) was used to validate the model. No statistically significant difference in age, sex, smoking status, histological type, or clinical stage was observed between the patients in the training and testing groups. The clinical and pathological characteristics of all patients are listed in Table I. Bead suspension was retrieved from a $4^{\circ} \mathrm{C}$ refrigerator, and inverted repeatedly to evenly resuspend the solution. To the 200- $\mu$ l sample tubes, $7 \mu$ of solid-phase extraction (SPE)-CM magnetic bead suspension, $10 \mu 1$ of serum, and $95 \mu 1$ of SPE-CB (Beijing Bioyong Technology Co., Ltd.) were added, and triturated several times to thoroughly mix the magnetic beads, SPE-CB and serum. The mixture was maintained at $21^{\circ} \mathrm{C}$ for $5 \mathrm{~min}$. Samples were placed in the magnetic bead separator for $1 \mathrm{~min}$ to separate the magnetic beads from the solution. After the liquid became clear, the remaining liquid was removed. Samples were removed from the magnetic bead separator and $100 \mu \mathrm{l}$ of SPE-CW (Beijing Bioyong Technology Co., Ltd.) was added to the sample, and triturated several times to mix the magnetic beads and SPE-CW thoroughly. The mixture was held at $21^{\circ} \mathrm{C}$ for $2 \mathrm{~min}$. Samples were placed in the magnetic bead separator for $1 \mathrm{~min}$ to separate the magnetic beads from the solution. When the liquid became clear, the remaining liquid was removed. The abovementioned step was repeated twice. Then, $10 \mu l$ of SPE-CE (Bioyong Tech) was added to each sample tube, and triturated 10 times to mix the magnetic beads and SPE-CE thoroughly. The mixture was held at $21^{\circ} \mathrm{C}$ for $5 \mathrm{~min}$. Samples were placed in the magnetic bead separator for $1 \mathrm{~min}$ to separate the magnetic beads from the solution. When the liquid became clear, it was transferred to a dry sample tube, and stored in a freezer at $-20^{\circ} \mathrm{C}$ for MS analysis.

Targeting. Saturated HCCA, $0.1 \%$ TFA and $50 \%$ ACN were mixed in an Eppendorf tube to form the matrix solution. The standard peptide mixture (polypeptide mixture) was mixed thoroughly in a 2:1 ratio. For external instrument calibration, $1 \mu \mathrm{l}$ of this sample was used. Next, $1 \mu \mathrm{l}$ of each sample after magnetic bead processing and $1 \mu \mathrm{l}$ of the prepared matrix solution were mixed thoroughly in a 1:1 ratio. Then, $1 \mu \mathrm{l}$ of this sample was placed on an MTP Anchorchip target, and allowed to air-dry at $21^{\circ} \mathrm{C}$.

MS analysis. After extracting serum peptides from the SPE-CM magnetic beads, linear mode detection via MALDI-TOF-MS was performed. After targeting, the MTP Anchorchip target was placed in a MALDI-TOF mass spectrometer for analysis. To reduce operational error and systematic error, one standard target (standardized peptide mixture) was used for each sample 
Table I. Basic information on the characteristics of patients with SCLC and healthy subjects.

A, Training group $(\mathrm{n}=108)$

\begin{tabular}{|c|c|c|c|}
\hline Characteristics & $\operatorname{SCLC}(n=54)$ & Healthy individual $(n=54)$ & P-value \\
\hline Age (years) & & & 0.894 \\
\hline Median & 59 & 56 & \\
\hline Range & $36-83$ & $31-72$ & \\
\hline Sex & & & 0.774 \\
\hline Male & 33 & 32 & \\
\hline Female & 21 & 22 & \\
\hline Ever smoked & & & 0.482 \\
\hline Yes & 28 & 24 & \\
\hline No & 26 & 30 & \\
\hline Staging & & & - \\
\hline LD & 5 & - & \\
\hline ED & 49 & - & \\
\hline
\end{tabular}

B, Testing group $(n=36)$

\begin{tabular}{|c|c|c|c|}
\hline Characteristics & $\operatorname{SCLC}(n=18)$ & Healthy individual $(n=18)$ & $\mathrm{P}$-value \\
\hline Age (years) & & & 0.673 \\
\hline Median & 57 & 55 & \\
\hline Range & $42-74$ & $35-68$ & \\
\hline Sex & & & 0.916 \\
\hline Male & 10 & 9 & \\
\hline Female & 8 & 9 & \\
\hline Ever smoked & & & 0.910 \\
\hline Yes & 10 & 8 & \\
\hline No & 8 & 10 & \\
\hline Staging & & & - \\
\hline LD & 0 & - & \\
\hline ED & 18 & - & \\
\hline
\end{tabular}

SCLC, small cell lung cancer; LD, limited disease; ED, extensive disease.

group, before analysis, as an external sample for external instrument calibration. The best scanning mass-to-charge ratio $(\mathrm{m} / \mathrm{z})$ range for MALDI-TOF-MS is $0.8-10 \mathrm{kDa}$. Each scan accumulated 500 mass spectrum signals, and each sample accumulated 3,000 signals. The accumulated spectra were saved, thereby generating an accurate serum peptide mass fingerprint map comprising peptide peaks with different $\mathrm{m} / \mathrm{z}$ ratios

Data collection and preservation. The datasets generated and/or analyzed during the current study are available in a repository hosted by Mendeley at the following link: http://dx.doi.org/10.17632/btg4dknchy.1, which includes the 'final upload data for this experiment', and whether the data uploaded onto Mendeley was used to establish the prediction models or validate the classification model, as well as its stability and reliability (10).
Statistical analysis. CPT software was used for the statistical analysis of all mass spectral data obtained. Before complete analysis using CPT, the original mass spectra of the samples were processed, including baseline correction, alignment and normalization using CPT. Next, the statistical analysis of the serum peptide spectra of the 54 patients with SCLC and 54 healthy individuals composing the training group was conducted using the statistical algorithms included in CPT. The peptides differentially expressed between the two groups were obtained; three algorithms [genetic algorithm (GA), supervised neural networks and quick classifier], which components of the CPT, were used to establish the prediction models.

Similarly, the statistical analysis of urine peptides revealed the urine peptides differentially expressed between the 54 patients with SCLC and 54 healthy individuals in the training group, and a urine classification model for SCLC 

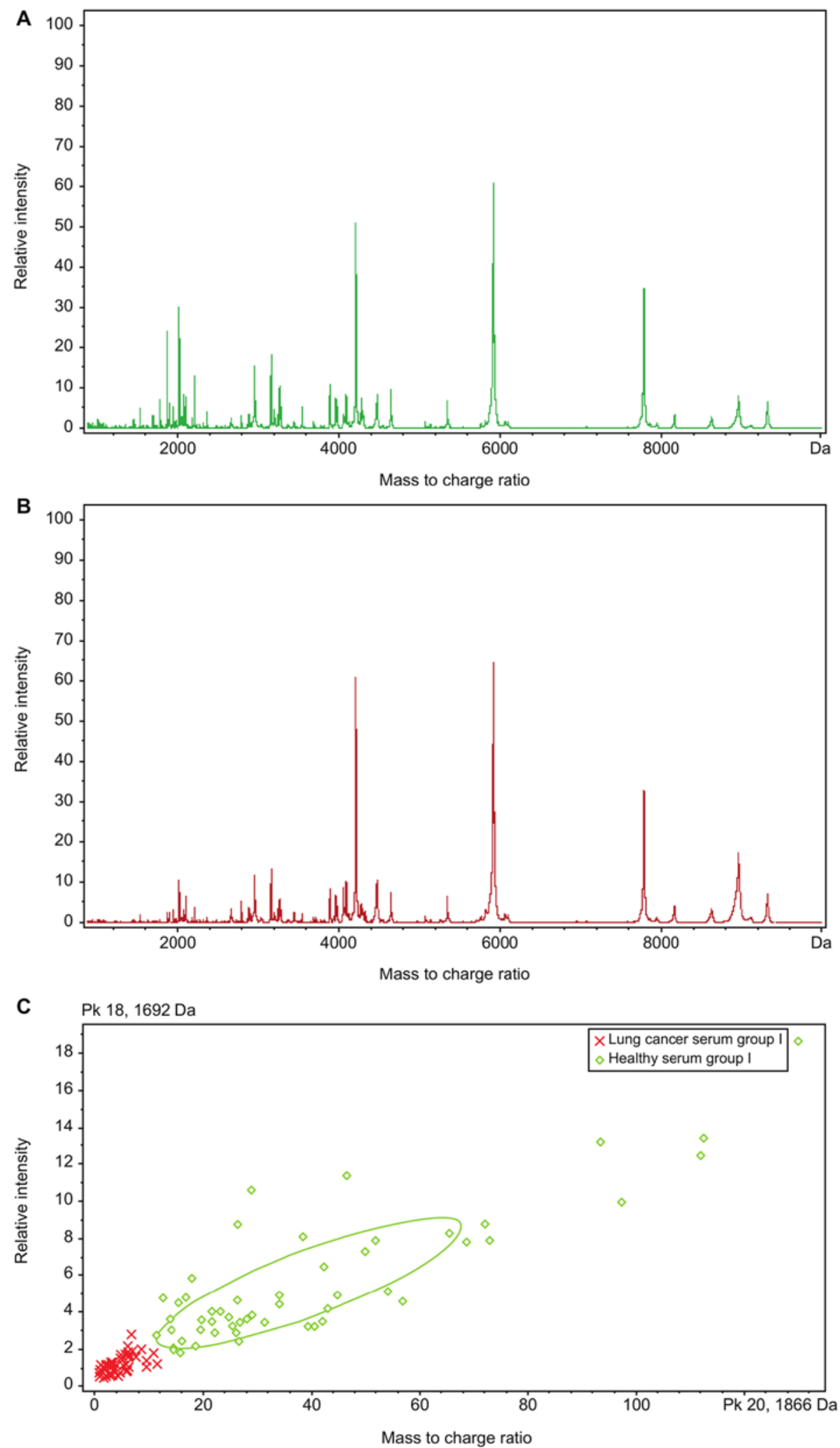

Figure 1. Serum peptide profiles of the training group. (A) Lung cancer serum group I and (B) healthy serum group. (C) Clustering analysis of MS-based serum peptide profiles (red, lung cancer serum group I; green, healthy serum group I). MS, mass spectrometry.

diagnosis was constructed from a number of peptides selected by CPT. The categorical variables were analyzed using the $\chi^{2}$ test; GA was used to identify differentially expressed peptides between the 54 SCLC patients and 54 healthy controls; Cross Tabulations analysis was used for sensitivity and specificity; at the same time, the plasma and urine classification models were combined to verify the specificity and sensitivity of their combinations. $\kappa$ test was used to perform consistency analysis. All statistical tests were two-tailed, and a P-value of $<0.0005$ was considered statistically significant. 
Table II. Differential peaks in serum from the patients with SCLC compared with healthy individuals in the training group.

\begin{tabular}{|c|c|c|c|c|}
\hline $\mathrm{m} / \mathrm{z}(\mathrm{Da})$ & $\begin{array}{l}\text { Peak areas of the } \\
\text { healthy group } \mathrm{I}(\mathrm{X} \pm \mathrm{S})\end{array}$ & $\begin{array}{l}\text { Peak areas of the } \\
\text { SCLC group I }(X \pm S)\end{array}$ & $\begin{array}{l}\text { Peak areas in patients with SCLC } \\
\text { compared with the healthy individuals }\end{array}$ & $\mathrm{P}$-value \\
\hline $1,866.37$ & $24.8 \pm 17.7$ & $2.85 \pm 1.61$ & $\downarrow$ & $<0.0005$ \\
\hline $1,779.22$ & $7.7 \pm 5.4$ & $1.19 \pm 0.59$ & $\downarrow$ & $<0.0005$ \\
\hline $1,692.13$ & $3.47 \pm 2.21$ & $0.77 \pm 0.33$ & $\downarrow$ & $<0.0005$ \\
\hline $3,541.28$ & $5.56 \pm 3.18$ & $2.61 \pm 1.46$ & $\downarrow$ & $<0.0005$ \\
\hline $5,251.47$ & $0.5 \pm 0.18$ & $0.87 \pm 0.32$ & $\uparrow$ & $<0.0005$ \\
\hline $2,210.62$ & $13.3 \pm 9.48$ & $4.03 \pm 4.15$ & $\downarrow$ & $<0.0005$ \\
\hline $2,366.84$ & $4.26 \pm 2.86$ & $1.45 \pm 1$ & $\downarrow$ & $<0.0005$ \\
\hline $1,968.41$ & $1.94 \pm 0.69$ & $1.28 \pm 0.34$ & $\downarrow$ & $<0.0005$ \\
\hline $8,952.22$ & $8.37 \pm 6.19$ & $16.59 \pm 9.9$ & $\uparrow$ & $<0.0005$ \\
\hline $4,055.64$ & $3.66 \pm 2.07$ & $9.41 \pm 6.81$ & $\uparrow$ & $<0.0005$ \\
\hline $2,082.49$ & $8.75 \pm 6.06$ & $3.56 \pm 2.34$ & $\downarrow$ & $<0.0005$ \\
\hline $3,444.06$ & $1.8 \pm 0.84$ & $2.96 \pm 1.44$ & $\uparrow$ & $<0.0005$ \\
\hline 839.2 & $4.38 \pm 1.87$ & $2.47 \pm 1.4$ & $\downarrow$ & $<0.0005$ \\
\hline $1,450.69$ & $2.61 \pm 1.8$ & $1.1 \pm 0.55$ & $\downarrow$ & $<0.0005$ \\
\hline $1,980.53$ & $2.46 \pm 0.96$ & $1.47 \pm 0.55$ & $\downarrow$ & $<0.0005$ \\
\hline $1,011.84$ & $2.53 \pm 1.7$ & $0.98 \pm 0.67$ & $\downarrow$ & $<0.0005$ \\
\hline $1,887.67$ & $2.68 \pm 1.47$ & $1.44 \pm 1.1$ & $\downarrow$ & $<0.0005$ \\
\hline $1,882.02$ & $2.56 \pm 1.81$ & $1.04 \pm 0.89$ & $\downarrow$ & $<0.0005$ \\
\hline $4,111.85$ & $1.45 \pm 0.53$ & $2.33 \pm 1.09$ & $\uparrow$ & $<0.0005$ \\
\hline
\end{tabular}

SCLC, small cell lung cancer; $\uparrow$, signals with a higher peak area in the SCLC group; $\downarrow$, signals with a lower peak area in the SCLC group.

All statistical tests were performed using SPSS software (version 19; IBM Corp.).

\section{Results}

Serum peptide mass fingerprinting map for training set. Fig. 1 shows the serum peptide mass fingerprinting maps of lung cancer serum group I and healthy serum group I obtained by performing MALDI-TOF-MS to detect serum peptides in the training set extracted using the magnetic beads for the 54 patients with SCLC and 54 healthy individuals, respectively, in the training group.

Analysis of differentially expressed peptides in serum samples of training set. The serum peptide mass fingerprinting maps of lung cancer serum group I and healthy serum group I were analyzed using CPT, which identified 126 peptide peaks in total; 19 peaks were significantly different $(\mathrm{P}<0.0005$; AUC $\geq 0.8$ ) between the 2 groups (Table II).

The clustering analytical method of CPT (Fig. 1C) was used for comparison analysis of the serum peptide mass fingerprinting maps of lung cancer serum group I and healthy serum group I, and GAs were obtained. The result of this algorithm showed that the optimal template comprised 5 peptides whose $\mathrm{m} / \mathrm{z}$ peptide peaks were at $0.8114,0.83425,1.86655,4.11133$ and $5.81192 \mathrm{kDa}$ (Fig. 2). The resulting model had a lung cancer detection rate of $99.04 \%$ and cross-validation rate of $91.47 \%$.

For the sera of the 18 patients with lung cancer and 18 healthy individuals in the testing set, the serum peptide mass fingerprinting map was similarly obtained using CPT, and the model constructed using GA was validated. The 18 patients with lung cancer and 18 healthy individuals were accurately identified ( 1 false negative among the 18 patients with lung cancer, 2 false positives among the 18 healthy individuals; the others were correctly identified). Blinded validation showed that the model had a specificity of $90 \%$ and sensitivity of $95 \%$.

Analysis of differentially expressed peptides in urine samples of training set. Fig. 3 shows the urine peptide mass fingerprinting maps of lung cancer urine group I and healthy urine group I, obtained using MALDI-TOF-MS to detect urine peptides in the training set extracted from the magnetic beads for the 54 patients with SCLC and 54 healthy individuals in the training group.

The urine peptide mass fingerprinting maps of lung cancer urine group I and healthy urine group I were analyzed using CPT, which identified 132 peptide peaks in total; 8 peaks were significantly different $(\mathrm{P}<0.0005 ; \mathrm{AUC} \geq 0.8)$ between the two groups (Table III).

The clustering analytical method of CPT (Fig. 3C) was used for comparison analysis of the urine peptide mass fingerprinting maps of lung cancer urine group I and healthy urine group I, and GAs were obtained. The result of this algorithm showed that the optimal template comprised 5 peptides whose $\mathrm{m} / \mathrm{z}$ peptide peaks were at 1.0724, 2.37692, 2.7554, 4.75475 and $4.7949 \mathrm{kDa}$ (Fig. 4). The resulting model had a lung cancer detection rate of $87.81 \%$ and cross-validation rate of $79.49 \%$.

For the urine samples of the 18 patients with lung cancer and 18 healthy individuals in the testing set, the urine peptide 

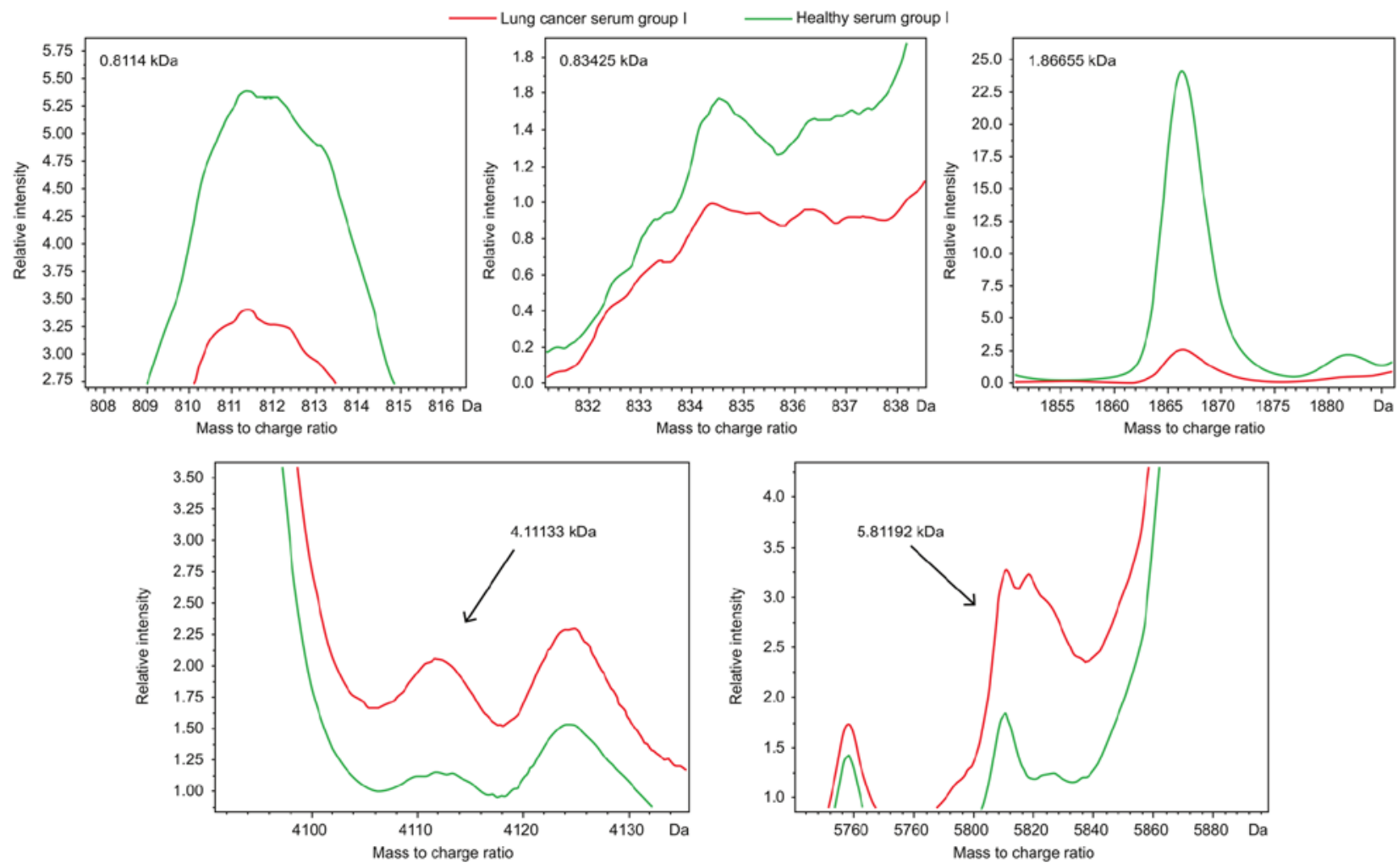

Figure 2. Specific peptide peaks of model profiles (red, lung cancer serum group I; green, healthy serum group I).

mass fingerprinting map was similarly obtained using CPT, and the model constructed using GA was validated. The 18 patients with lung cancer and 18 healthy individuals were accurately identified ( 2 false negatives among the 18 patients with lung cancer, 4 false positives among the 18 healthy individuals; the others were correctly identified). Blinded validation showed that the model had a specificity of $80 \%$ and a sensitivity of $90 \%$.

Peptide peak identification. To further study the specific peptide peaks for serum and urine, 3 peptide peaks with simultaneously upregulated expression in the sera and urine of the patients with SCLC were examined. Their $\mathrm{m} / \mathrm{z}$ ratios were $2.3764,0.8778$ and $0.8616 \mathrm{kDa}$. A search of the liquid chromatography/MS secondary mass spectrum database of our laboratory determined the $2.3764-\mathrm{kDa}$ peptide peak to correspond to fibrinogen $\alpha$, having the sequence SYKMADEAG SEADHEGTHSTKRGHAKSRPV, the $0.8778-\mathrm{kDa}$ peptide peak to correspond to glucose-6-phosphate isomerase, having the sequence RNGFKSHALQLNNRQI, and the 0.8616-kDa peptide peak to correspond to cyclin-dependent kinase-1, having the sequence SSKITHRIHWESASLLR.

Consistency analysis of serum model and urine model blind sample verification results. The results of the blind test results of the serum model and the urine model were further analyzed. It was found that in 18 patients with lung cancer and 18 healthy individuals, in the serum model, 1 patient with lung cancer was false negative, and this patient gave the same result in the urine model. Of the 2 patients who showed false positive results, 1 was still a false positive in the urine model, and the other was diagnosed correctly to be a healthy individual. The remaining patients showing false positive or false negative results in the urine model were diagnosed correctly in the serum model. It can be seen that the sensitivity and specificity of the serum model are higher than those of the urine model, and the serum model is in good agreement with the urine model $(\kappa=0.720$; $\mathrm{P}<0.001)$. The results of the blind sample verification results are shown in Table IV.

\section{Discussion}

Mass spectrometry analysis is an important detection method in cancer proteomics. Currently, the mass spectrometric techniques common in biology include MALDI-TOF-MS, electrospray ionization (ESI)-MS, and surface-enhanced laser desorption/ionization (SELDI)-TOF-MS. High tolerance to salt and impurities and high sensitivity render MALDI-TOF-MS superior to other MS techniques (11). After reflective and delayed extraction techniques were introduced for the method, the resolution of the method increased greatly, reaching $\leq 10 \mathrm{ppm}$ for detecting small molecular peptides of $\sim 2 \mathrm{kDa}(12,13)$.

Considering the high sensitivity, high throughput and other advantages of MS for protein identification, numerous studies have attempted to use MS detection for early-stage cancer diagnosis, primarily via MS analysis of samples from patients with cancer and healthy individuals, thereby discovering differentially expressed cancer-specific proteins or peptides. For example, Monari et al (14) used IMAC30-Cu and H50 SELDI-TOF-MS chips to study 44 patients with lung cancer and 19 healthy individuals; the study observed 28 significantly different peaks and constructed a model. The sensitivity and specificity of the IMAC30-Cu chip were 70.45 and $68.42 \%$, respectively, and the sensitivity and specificity of the H50 chip 

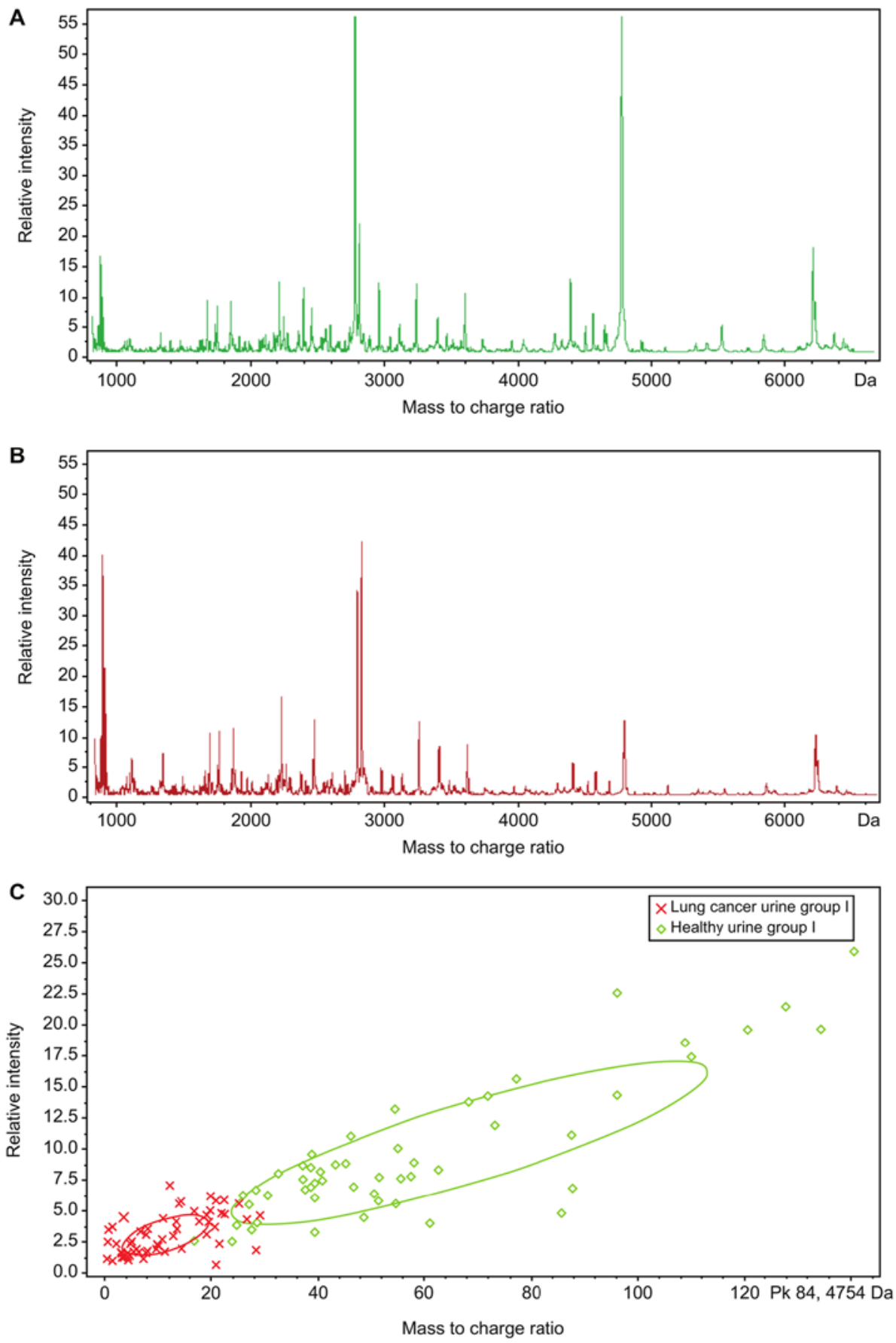

Figure 3. Urine peptide profiles of training group. (A) Lung cancer urine group I and (B) healthy urine group I. (C) Clustering analysis of MS-based urine peptide profiles (red, lung cancer urine group I; green, healthy urine group I). MS, mass spectrometry.

were 72.73 and $73.68 \%$, respectively. Han et al (15) used metal affinity protein chips and SELDI-TOF technology to study serum samples and used 3 proteins to construct a classification tree model. Blinded validation revealed a sensitivity of $89 \%$, specificity of $91 \%$, and accuracy of $90 \%$. Hocker et al (16) used ESI-MS to analyze serum samples of 43 patients with early-stage lung cancer and 21 healthy individuals, and the method using the differentially expressed proteins identified for differentiating between the cancer patients and healthy individuals had an overall efficacy of $80 \%$ and sensitivity of $84 \%$. Results from these studies demonstrate that MS detection methods can recognize cancer-related molecules contained in the sera of patients with lung cancer, and can potentially be used for early lung cancer diagnosis.

However, the sensitivity and specificity of blinded validation in the aforementioned studies were relatively low, with no further validation being subsequently performed for the following reasons: i) All traditional methods for extracting proteins and peptides from samples, including pre-processing techniques, such as 2-dimensional gel electrophoresis, protein chip-based methods, or solid phase extraction, have operational complexities and low extraction efficiencies, resulting in the loss of usable information; and ii) ESI, SELDI-TOF-MS, and other mass spectrometric detection 
Table III. Differential peaks in urine from the patients with SCLC compared with healthy individuals in the training group.

\begin{tabular}{|c|c|c|c|c|}
\hline $\mathrm{m} / \mathrm{z}(\mathrm{Da})$ & $\begin{array}{l}\text { Peak areas of the } \\
\text { healthy group I }(X \pm S)\end{array}$ & $\begin{array}{l}\text { Peak areas of the } \\
\text { SCLC group I }(X \pm S)\end{array}$ & $\begin{array}{c}\text { Peak areas in SCLC patients } \\
\text { compared with the healthy individuals }\end{array}$ & P-value \\
\hline $4,754.75$ & $0.5 \pm 0.18$ & $0.87 \pm 0.32$ & $\downarrow$ & $<0.0005$ \\
\hline $2,376.92$ & $4.26 \pm 2.86$ & $1.45 \pm 1.00$ & $\downarrow$ & $<0.0005$ \\
\hline $4,794.90$ & $0.61 \pm 0.19$ & $0.85 \pm 0.26$ & $\downarrow$ & $<0.0005$ \\
\hline $4,712.28$ & $1.8 \pm 0.84$ & $2.96 \pm 1.44$ & $\downarrow$ & $<0.0005$ \\
\hline $4,625.74$ & $9.98 \pm 3.28$ & $7.45 \pm 2.76$ & $\downarrow$ & $<0.0005$ \\
\hline $4,900.42$ & $2 \pm 0.92$ & $4.47 \pm 4.54$ & $\downarrow$ & $<0.0005$ \\
\hline $6,341.19$ & $5.95 \pm 2.33$ & $3.6 \pm 1.8$ & $\uparrow$ & $<0.0005$ \\
\hline $2,755.40$ & $2.93 \pm 1.25$ & $1.9 \pm 0.95$ & $\downarrow$ & $<0.0005$ \\
\hline
\end{tabular}

SCLC, small cell lung cancer; $\uparrow$, signals with a higher peak area in the SCLC group; $\downarrow$, signals with a lower peak area in the SCLC group.
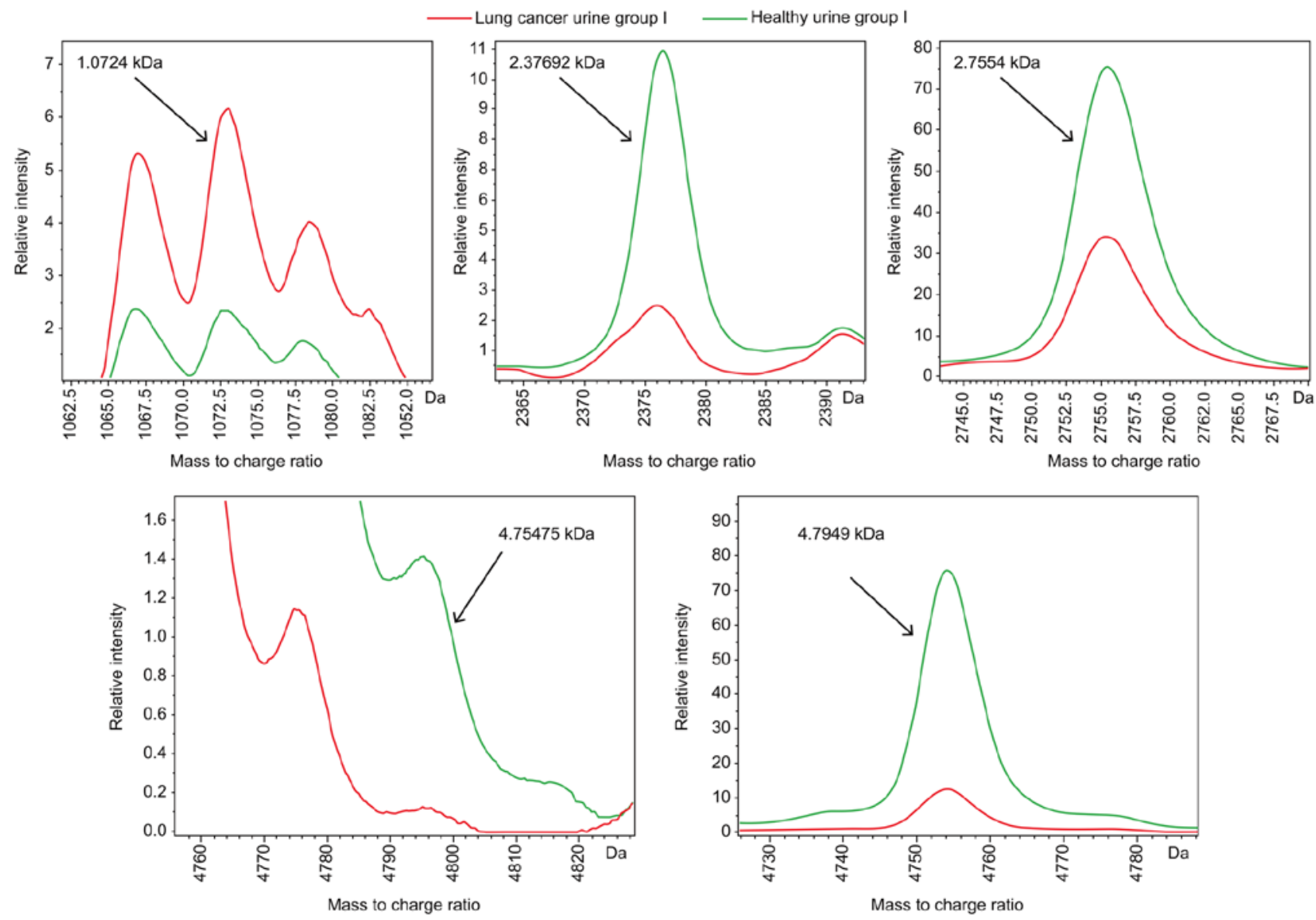

Figure 4. Specific peptide peaks of model (red, lung cancer urine group I; green, healthy urine group I).

methods have low sensitivity and resolution, and reflect limited information. These limitations have restricted their application in disease-specific marker discovery and clinical diagnosis. Hence, the present study used the CPT detection system developed by Bruker Daltonics (17). It includes a magnetic bead separation system, a MALDI-TOF-MS system, analytical software and automated body fluid sample processing system. Its advantages are that it contains the latest Anchor-Chip patented sample targeting technology, which can greatly increase the analyte concentration, and increase sensitivity to 10-100 times that of conventional chips, and the resolution, repeatability and reproducibility of MALDI-TOF-MS are higher than those of SELDI-TOF and other mass spectrometric techniques (18). At various world-class hospitals and medical research institutes, this technology has been widely used in studies on the early diagnosis of ovarian cancer, lung adenocarcinoma and multiple myeloma patients (19-21). 
Table IV. Consistency analysis of serum model and urine model blind sample verification results.

\begin{tabular}{lrrrrr}
\hline & \multicolumn{2}{c}{ Serum model } & & & \\
\cline { 2 - 4 } Urine model & SCLC & Healthy & Total & $\kappa$ & P-value \\
\hline SCLC & 16 & 4 & 20 & 0.720 & $<0.001$ \\
Healthy & 3 & 13 & 16 & & \\
Total & 19 & 17 & 36 & & \\
\hline
\end{tabular}

SCLC, small cell lung cancer.

The present study used the CPT detection system for the sera and urine of patients with SCLC and healthy individuals, and discovered 19 differentially expressed peptides in serum and 8 differentially expressed peptides in urine. Next, the statistical software screened 5 peptide peaks for constructing the serum and urine SCLC diagnostic classification models. Blinded model validation was performed, and satisfactory results were obtained, demonstrating that the classification model of the present study had a certain theoretical value for identifying patients with SCLC, although it still requires further clinical validation. The classification model of the present study was unlike traditional diagnostic models based on a single index for detection; rather, it used a group of peptide indicators as detection criteria. It used biological mass spectrometry as the support platform, and bioinformatics as the bridge for peptide research, and via changes in the blood peptidome of patients with lung cancer, specific cancer-related information could be obtained to establish cancer diagnosis. When blood flows through the tumor microenvironment, it may cause various slight changes in unknown components of serum proteins/peptides, making the use of markers in combination more effective than the use of a single marker. In the present study, the statistically significantly differentially expressed proteins observed in both serum and urine were fibrinogen $\alpha$, glucose-6-phosphate isomerase and cyclin-dependent kinase-1. Fibrinogen is a protein with coagulant activity synthesized by the liver; a number of studies have shown that fibrinogen is upregulated in cancers of the urinary system, reproductive system and gastrointestinal tract (22-24).

The present study identified high fibrinogen $\alpha$ expression in the sera and urine of patients with SCLC, which may be associated with the hypercoagulable state of patients with cancer. Glucose-6-phosphate isomerase is an important enzyme in humans. The main function is to catalyze the exchange between D-glucose-6-phosphate and D-fructose-6-phosphate, which is an important enzyme for glycolysis and gluconeogenesis. Although there are no reports on its involvement in cancer development and progression, to the best of the authors' knowledge. Cyclin-dependent kinase 1 is a protein kinase family member, and depends on binding with cell cycle proteins to serve key roles in the systematic progress of the cell cycle. When cell cycle proteins are lacking, or cyclin-dependent kinase inhibitors are present, cell division is arrested and cell death may occur (25). Future studies will employ ELISA to validate the expression of the three peptides identified in the serum and urine samples of the patients with SCLC. The majority of SCLC cases are already in LD stages when discovered (there were only 5 ED patients in the present study). The model has been established and validated in patients with LD SCLC, but whether this model can be used for ED SCLC patient diagnosis remains to be verified. The future aims of this study are to increase the number of LD SCLC samples used, validate the reproducibility and reliability of the diagnostic classification model, further identify the peptides corresponding to the $\mathrm{m} / \mathrm{z}$ peaks, and evaluate protein/peptide expression, to identify novel tumor markers of lung cancer, and study their associations with the molecular mechanisms of lung cancer development.

The use of the specific serum and urine peptide-spectrum model for SCLC was revealed to be accurate in a small cohort of patients. In the future, a large-scale validation with actual clinical operation should be conducted, and it is expected to create the foundation for developing a serum- and urine-based diagnosis that is rapid, non-invasive and requires a small sample quantity.

The present study selected the sera and urine of patients with SCLC and healthy individuals as the research subjects, used MALDI-TOF-MS with the CPT system to detect serum and urine peptides, performed small-scale validation of specific SCLC-related peptides, and achieved high sensitivity and specificity, as the samples originated primarily from LD-stage patients. Samples from more ED-stage patients, as well as dynamic monitoring of samples from high-risk populations, should be included in future studies, to further validate the sensitivity and specificity of this diagnostic model.

\section{Acknowledgements}

Not applicable.

\section{Funding}

This study was funded by the Chinese National Instrumentation Program (Beijing, China; grant no. 2011YQI70067).

\section{Availability of data and materials}

The datasets generated and/or analyzed during the current study are available in a repository hosted by Mendeley at the following link: http://dx.doi.org/10.17632/btg4dknchy.1.

\section{Authors' contributions}

XLiu, ZL and PL conceived and designed this study. KH and BX acquired the data. PL, CT, SY and HQ analyzed and interpreted the data. The original draft was written, and the subsequent review and editing for important intellectual content was performed by HG, PL and XLi.

\section{Ethics approval and consent to participate}

This study was conducted using protocols approved by the ethics committee of The Fifth Medical Centre, Chinese PLA General Hospital for drug clinical trials (no. 2012-11-171). All patients provided written informed consent before undergoing 
any treatment or serum or urine sampling. Patients with heart, liver, kidney, or other major organ diseases were excluded.

\section{Patient consent for publication}

Not applicable.

\section{Competing interests}

The authors declare that they have no competing interests.

\section{References}

1. Siegel R, Ma J, Zou Z and Jemal A: Cancer statistics, 2014. CA Cancer J Clin 64: 9-29, 2014.

2. Ferlay J, Soerjomataram I, Ervik M, Dikshit R, Eser S, Mathers C, Rebelo M, Parkin DM, Forman D and Bray F: GLOBOCAN 2012 v1.0, Cancer incidence and mortality worldwide: IARC cancer base no. 11 [Internet]. International Agency for Research on Cancer, Lyon, 2014.

3. Kahnert K, Kauffmann-Guerrerom D and Huber RM SCLC-state of the art and what does the future have in store? Clin Lung Cancer 17: 325-333, 2016.

4. National Central Cancer Registry of China. China Cancer Registration Annual Report, 2014.

5. Evans WK, Flanagan WM, Miller AB, Goffin JR, Memon S, Fitzgerald N and Wolfson MC: Implementing low-dose computed tomography screening for lung cancer in Canada: Implications of alternative at-risk populations, screening frequency, and duration. Curr Oncol 23: e179-e187, 2016.

6. Yeh CY, Adusumilli R, Kullolli M, Mallick P, John EM and Pitteri SJ: Assessing biological and technological variability in protein levels measured in pre-diagnostic plasma samples of women with breast cancer. Biomark Res 17: 30, 2017.

7. Xu W, Li X, Lin N, Zhang X, Huang X, Wu T, Tai Y, Chen S, $\mathrm{Wu} \mathrm{CH}$, Huang $\mathrm{M}$ and Wu S: Pharmacokinetics and tissue distribution of five major triterpenoids after oral administration of Rhizoma Alismatis extract to rats using ultra high-performance liquid chromatography-tandem mass spectrometry. J Pharm Biomed Anal 146: 314-323, 2017.

8. Popp R, Li H, Le Blanc A, Mohammed Y, Aguilar-Mahecha A, Chambers AG,Lan C,PoetzO,Basik M, BatistGand Borchers CH: Immuno-matrix-assisted laser desorption/ionization assays for quantifying AKT1 and AKT2 in breast and colorectal cancer cell lines and tumors. Anal Chem 89: 10592-10600, 2017.

9. National Comprehensive Cancer Network: NCCN Clinical Practice Guidelines in Oncology-Non-Small Cell Lung Cancer, version 2.2016. http://www.nccn.org/professionals/physician_ gls/f_guidelines. Asp. Accessed December 04, 2015.

10. Panpan IV: Exploratory study on application of MALDI-TOF-MS to detect serum and urine peptides related to small cell lung carcinoma. Mendeley Data, v1, 2018. http://dx.doi.org/10.17632/btg4dknchy.1

11. Cho A and Normile D: Nobel prize in chemistry: Mastering macromolecules. Science 298: 527-528, 2002.

12. Dekker LJ, Boogerd W, Stockhammer G, Dalebout JC, Siccama I, Zheng P, Bonfrer JM, Verschuuren JJ, Jenster G, Verbeek MM, et al: MALDI-TOF mass spectrometry analysis of cerebrospinal fluid tryptic peptide profiles to diagnose Leptomeningeal metastases in patients with breast cancer. Mol Cell Proteomics 4: 1341-1349, 2005.
13. Villanueva J, Shaffer DR, Philip J, Chaparro CA, ErdjumentBromage H, Olshen AB, Fleisher M, Lilja H, Brogi E, Boyd J, et al: Differential exoprotease activities confer tumor-specific serum peptidome patterns. J Clin Invest 116: 271-284, 2006.

14. Monari E, Casali C, Cuoghi A, Nesci J, Bellei E, Bergamini S, Fantoni LI, Natali P, Morandi U and Tomasi A: Enriched sera protein profiling for detection of non-small cell lung cancer biomarkers. Proteome Sci 9: 55, 2011.

15. Han KQ, Huang G, Gao CF, Wang XL, Ma B, Sun LQ and Wei ZJ: Identification of lung cancer patients by serum protein profiling using surface-enhanced laser desorption/ionization time-of-flight mass spectrometry. Am J Clin Oncol 31: 133-139, 2008.

16. Hocker JR, Peyton MD, Lerner MR, Mitchell SL, Lightfoot SA, Lander TJ, Bates-Albers LM, Vu NT, Hanas RJ, Kupiec TC, et al: Serum discrimination of early-stage lung cancer patients using electrospray-ionization mass spectrometry. Lung Cancer 74: 206-211, 2011.

17. Ketterlinus R, Hsieh SY, Teng SH, Lee H and Pusch W: Fishing for biomarkers: Analyzing mass spectrometry data with the new ClinProTools software. Biotechniques 38 (Suppl): S37-S40, 2009.

18. Telesmanich NR, Agafonova VV, Chemisova OS, Chaĭka IA, Vodop'ianov AS, Goncharenko EV and Telicheva VO: Proteome mass-spectrometric analysis and typing of Vibrio cholerae strains isolated in the territory of Russian Federation in 2010-2012. Zh Mikrobiol Epidemiol Immunobiol: 97-101, 2014 (In Russian).

19. Qiu F, Liu HY, Dong ZN, Feng YJ, Zhang XJ and Tian YP: Searching for potential ovarian cancer biomarkers with matrix-assisted laser desorption/ionization time-of-flight mass spectrometry. Am J Biomed Sci 1: 80-90, 2009.

20. Xu J, Xu B, Tang C, Li X, Qin H, Wang W, Wang H, Wang Z, $\mathrm{Li} \mathrm{L,} \mathrm{Li} \mathrm{Z,} \mathrm{et} \mathrm{al:} \mathrm{The} \mathrm{exploration} \mathrm{of} \mathrm{peptide} \mathrm{biomarkers} \mathrm{in}$ malignant pleural effusion of lung cancer using Matrix-assisted laser desorption/ionization time-of-flight mass spectrometry. Dis Markers 2017: 3160426, 2017.

21. Deulofeu M, Kolářová L, Salvadó V, María Peña-Méndez E, Almáši M, Stork M, Pour L, Boadas-Vaello P, Ševčíková S, Havel $J$ and Vaňhara P: Rapid discrimination of multiple myeloma patients by artifcial neural networks coupled with mass spectrometry of peripheral blood plasma. Sci Rep 9: 7975, 2019.

22. Coverley D, Higgins G, West D, Jackson OT, Dowle A, Haslam A, Ainscough E, Chalkley R and White J: A quantitative immunoassay for lung cancer biomarker CIZ1b in patient plasma. Clin Biochem 50: 336-343, 2016

23. Kim YS, Gu BH, Choi BC, Kim MS, Song S, Yun JH, Chung MK, Choi CH and Baek KH: Apolipoprotein A-IV as a novel gene associated with polycystic ovary syndrome. Int J Mol Med 31: 707-716, 2013.

24. Wu C, Luo Z, Tang D, Liu L, Yao D, Zhu L and Wang Z: Identification of carboxyl terminal peptide 410 of fibrinogen as a potential serum biomarker for gastric cancer. Tumour Biol 37: 6963-6970, 2016.

25. Parrilla A, Cirillo L, Thomas Y, Gotta M, Pintard L and Santamaria A: Mitotic entry: The interplay between Cdk1, Plk1 and Bora. Cell Cycle 15: 3177-3182, 2016.

This work is licensed under a Creative Commons Attribution-NonCommercial-NoDerivatives 4.0 International (CC BY-NC-ND 4.0) License. 